\title{
Safety of polyethylene glycol 3350 solution in chronic constipation: randomized, placebo-controlled trial
}

This article was published in the following Dove Press journal:

Clinical and Experimental Gastroenterology

15 July 2016

Number of times this article has been viewed

\author{
Thomas McGraw \\ Global Medical Affairs, Merck \& Co., \\ Inc., Kenilworth, NJ, USA
}

Purpose: To evaluate the safety and tolerability of aqueous solution concentrate (ASC) of polyethylene glycol (PEG) 3350 in patients with functional constipation.

Patients and methods: The patients who met Rome III diagnostic criteria for functional constipation were randomized in this multicenter, randomized, placebo-controlled, single-blind study to receive once daily dose of PEG 3350 (17 g) ASC or placebo solution for 14 days. The study comprised a screening period (visit 1), endoscopy procedure (visits 2 and 3), and followup telephone calls 30 days post-treatment. Safety end points included adverse events (AEs), clinical laboratory evaluations, vital signs, and others. The primary end points were the proportion of patients with abnormalities of the oral and esophageal mucosa, detected by visual and endoscopic examination of the oral cavity and esophagus, respectively, compared with placebo. A secondary objective was to compare the safety and tolerability of ASC by evaluating AEs or adverse drug reactions.

Results: A total of 65 patients were enrolled in this study, 31 were randomized to PEG 3350 ASC and 34 were randomized to placebo, of which 62 patients completed the study. No patients in either group showed abnormalities in inflammation of the oral mucosa during visit 2 (before treatment) or visit 3 (after treatment). Fewer abnormalities of the esophageal mucosa were observed in the PEG 3350 ASC group than in the placebo group on visit 3, with no significant difference in the proportion of abnormalities between the treatment groups. Overall, 40 treatment-emergent AEs were observed in $48.4 \%$ of patients treated with PEG 3350 ASC, and 41 treatment-emergent AEs were observed in $55.9 \%$ of patients treated with placebo - nonsignificant difference of $-7.5 \%$ (95\% CI: $-21.3,6.3)$ between treatment groups. No serious AEs or deaths were reported, and no patient discontinued because of an $\mathrm{AE}$.

Conclusion: PEG 3350 ASC is safe and well tolerated in patients with functional constipation (NCT01885104).

Keywords: polyethylene glycols, drug tolerance, constipation, laxatives, solutions

\section{Introduction}

Prevalence of chronic constipation in North America ranges from $2 \%$ to $27 \%$ of the adult population, with a higher incidence seen in the elderly (ratio, 1.5:1 versus young) and women (ratio, 2.2:1 versus men). ${ }^{1,2}$ A recent meta-analysis conducted in studies published through December 2010, reporting the prevalence of constipation, revealed a pooled global prevalence of $14 \%$ for constipation. ${ }^{3}$ Variation in the incidence of constipation exists due to several reasons such as differences in constipation definitions, whether constipation is physician or patient reported, and the population
Correspondence: Thomas McGraw Clinical Research \& PM Consulting LLC, 928 Long Hill Road, Millington, NJ 07946-023I, USA

Tel + I 9086044202

Email thomas_P_mcgraw@yahoo.com 
surveyed. ${ }^{2,4}$ Constipation rates are higher in patient-reported cases, wherein the definition of constipation also includes the symptoms, versus physician-reported cases, which are solely based on frequency of bowel movements (BM). ${ }^{2}$ Thus, to account for subjective and objective definitions of constipation, the globally accepted Rome III criteria for functional constipation were devised..$^{5}$ To meet the criteria, at least two of the following in $\geq 25 \%$ of defecations must be present: straining, lumpy or hard stools, sensation of incomplete evacuation, sensation of anorectal obstruction/blockage, manual maneuvers to facilitate defecation, and fewer than three defecations per week. In addition, loose stools must be rarely present without the use of laxatives, and there should be insufficient criteria for irritable bowel syndrome. These criteria must be fulfilled for 3 months, with symptom onset at least 6 months before diagnosis.

Pharmacological intervention with laxatives is frequently used to treat constipation. ${ }^{2}$ A multinational survey showed that in the US, $40 \%$ of adults with self-defined constipation used laxatives for treatment. ${ }^{2}$ Over-the-counter (OTC) laxatives used to manage constipation include polyethylene glycol (PEG) 3350 and lactulose, with an osmotic effect; psyllium, methylcellulose, and polycarbophil, which are bulk forming; and stimulant laxatives, such as senna and bisacodyl. ${ }^{4}$ In 1999, the US Food and Drug Administration (FDA) approved PEG 3350 (MiraLAX ${ }^{\text {TM} ; ~ B a y e r ~ A G, ~ L e v e r k u s e n, ~ G e r m a n y) ~}$ for the treatment of occasional constipation; in 2006, the FDA approved its OTC use (dosing instructions: a single dose of $17 \mathrm{~g}$ PEG 3350 dissolved in 4-8 ounces of water or liquid) based on multiple randomized controlled trials and pharmacokinetic studies. A single dose of PEG 3350, when taken for 7 days, was poorly absorbed (range, $0.15 \%-0.58 \%$, excreted in urine), and the majority of the study drug $(\sim 93 \%$ over 240 hours) was recovered in feces. ${ }^{6,7}$ Characterization of PEG 3350 concentration in human plasma, after single and multiple doses, revealed minimal systemic exposure. The drug levels in plasma were unquantifiable in most patients at 18-24 hours, with peak levels occurring at 2-4 hours, after which levels declined; half-life was 4-6 hours. ${ }^{7}$

PEG is an odorless, tasteless, nonmetabolizable, nonabsorbable polymer in powder form that is not fermented by bacterial flora. ${ }^{8}$ The laxative effect of PEG 3350 is due to sequestration of water in the lumen of the intestine, thereby increasing osmotic pressure. ${ }^{9}$ It has been traditionally used in large volumes as a gastrointestinal (GI) lavage solution for bowel cleansing before colonoscopy without harmful effects. Its efficacy and safety have been established in multiple clinical trials involving children and adults, including women, elderly, and severely intellectually and physically disabled patients with chronic constipation. ${ }^{10-18}$ PEG 3350 increased the number of BM per week, improved stool consistency, reduced time to first $\mathrm{BM}$, and provided significant relief from straining compared with placebo. ${ }^{10-16}$ Furthermore, PEG 3350 was generally safe and well tolerated in several short-term (72 hours to 4 weeks) $)^{15,16,19}$ and long-term (6 months to a year) ${ }^{11,14}$ studies. Adverse events (AEs) were minimal and comparable to placebo.

A novel, more convenient, OTC aqueous solution concentrate (ASC) of PEG 3350, which requires dilution of the concentrate in an appropriate volume (4-8 ounces) before ingestion, is in development. The diluted solution would provide the same final dose of PEG 3350 as the original powder formulation and is expected to provide identical clinical benefit in the relief of constipation. This study was conducted to address concerns regarding the safety and tolerability of ingesting undiluted PEG 3350 ASC, specifically focusing on oral and esophageal mucosa.

\section{Patients and methods Study design}

This multicenter, randomized, placebo-controlled, singleblind study (protocol \# CL2012-13, NCT01885104) was conducted at three clinical sites in the US in compliance with good clinical practice and with all applicable federal, state, and local laws, rules, and regulations, including the International Conference on Harmonization guidelines, and was consistent with the Declaration of Helsinki. This study was reviewed and approved by Schulman Associates IRB before start of study. Patients' informed consent forms were reviewed by the IRB and Merck Care. Written informed consent was obtained from all patients involved in the study.

The study comprised one screening period (visit 1), two in-house stays (visits 2 and 3), and two follow-up telephone calls (Figure 1). Patients were allowed to continue their usual laxative regimen between visit 1 and visit 2 . At visit 2 , eligibility for randomization was determined, and patients had to discontinue all laxatives during the 14-day treatment period. All qualified patients were required to remain at the site a day before the baseline upper GI endoscopy procedure to $\sim 24$ hours after endoscopy. Patients underwent an examination of the oral cavity and endoscopy according to the endoscopy site's standard operating procedures. Patients fasted for $\sim 10$ hours and had no fluids for $\sim 4$ hours before the baseline upper GI endoscopy procedure. Approximately 24 hours after the endoscopy, at 8 am, the study drug was dispensed to patients at the site. Rescue medications (bisacodyl $5 \mathrm{mg}$ ) and 


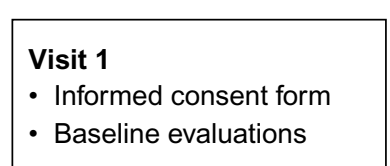

Up to
28 days

Visit 2

- Randomization

- Endoscopy

- Dispense treatment and diary

Figure I Study design.

Notes: ${ }^{a} \mathrm{~A}$ midtreatment phone call follow-up will be made to each subject to assess AEs $\sim 7$ days after the first dose.

Abbreviation: $A E$, adverse event.

diaries with instructions for completion were dispensed, and randomized patients were released from the site with either PEG 3350 or placebo. Patients were instructed to continue taking the treatment in the morning at home. Approximately 7 days after the subject had taken the test drug or placebo, a follow-up telephone interview assessed any possible safety and tolerance concerns. The time of dosing, problems with tolerance, use of concomitant medications, and occurrence of any AEs were recorded daily in the subject's diary. At the end of the 14-day treatment period, patients returned to the study site for visit 3 , which was $3 \pm 2$ days after completion of the treatment period. Examination of the oral cavity and an upper GI endoscopy procedure were performed the following morning, and patients were released from the site 24 hours later. The study was concluded with a telephone interview to collect AEs $\sim 30$ days after the end of the treatment. Patients with a serious AE (SAE) were evaluated with appropriate follow-up by the site. The subject was considered to have completed the study either after completion of the last visit or contact or after the last dose of the study treatment, whichever was later. Total study duration was $\sim 75$ days.

\section{Patients/participants}

Ambulatory adults aged 18-75 years who met Rome III diagnostic criteria for functional constipation ${ }^{5}$ were included in the study. Other key inclusion criteria were as follows: participants had to be healthy, other than functional constipation, with no history or evidence of any condition that might confound results of the study or might interfere with the patient's participation for the duration of the study, and patients had to agree not to use laxatives other than PEG 3350 or disallowed-concomitant medications for the duration of the study. Key exclusion criteria included abnormal baseline endoscopy and/or oral examination, medical history of impaired or difficulty swallowing foods and liquids, or suspected significant GI disease, severe or unexplained abdominal pain, allergy or intolerance to any of the study treatments, and renal impairment (estimated glomerular filtration rate, $<60 \mathrm{~mL} / \mathrm{min} / 1.73 \mathrm{~m}^{2}$ ) calculated using serum creatinine levels.

\section{Randomization and treatments}

Patients were randomized to receive PEG 3350 (17 g) ASC or placebo aqueous solution according to a computer-generated randomization schedule. These were administered once daily, in $\sim 30 \mathrm{~mL}$ (as measured in a provided dosing cup) at $\sim 8 \mathrm{am}$ each morning for 14 days. Patients were provided bisacodyl laxative tablets (5 mg, 1-3 tablets in a single daily dose according to label) to use as rescue medication if the patient had no BM for 72 hours after beginning the treatment phase of the study. Patients were allowed to take any medications not identified as excluded medication or not contraindicated for PEG 3350. The use of any laxative for the treatment of constipation, or medications known to cause constipation, was prohibited between visit 1 and visit 3 . Excluded medications included antibiotics associated with esophageal injury, other nonsteroidal anti-inflammatory drugs, corticosteroids and bisphosphonates, and medications associated with protection from esophageal injury such as proton pump inhibitors, antacids, alginic acid, and bicarbonate.

\section{Blinding}

The study was single-blinded (investigators and gastroenterologists) since the placebo aqueous solution was not identical to the PEG 3350 ASC.

\section{Outcome measures}

The primary end points were 1) the proportion of patients in each treatment group with inflammation (ie, redness, swelling, erosions, and ulceration) of the oral mucosa 
(ie, oral tissue, tongue, and teeth) as visually observed by a qualified gastroenterologist using the 0-3 Likert scale: 0 , no inflammation (no erythema and no erosion/ulceration); 1, mild inflammation (erythema without erosion/ulceration); 2 , moderate inflammation (erythema with erosion); and 3 , severe inflammation (erythema with ulceration), where erythema was clear abnormal reddening of the mucosa compared with a normal examination, erosion was a small superficial defect in the mucosa with a flat edge, and ulceration was a large and deeper excavated lesion with perceptible depth and a white base. 2) The proportion of patients in each treatment group with abnormalities (ie, erythema and erosion/ulceration) of the esophageal mucosa detected by endoscopic examination of the esophagus using the 0-3 Likert scale. Standard modern diagnostic endoscopy equipment in current use at the patient care facility of the clinical sites with state-of-the-art diagnostic photographic/ video documentation capability was used to document the esophageal endoscopic examination. A satisfactory quality recording with a sharp image was required during the procedure. A central reader, a gastroenterologist experienced in endoscopy and distinct from the gastroenterologist who performed the initial endoscopy, evaluated the esophageal mucosa by review of photographic images taken during endoscopy. The central reader was unaware of the study design, the hypothesis, the subjects' clinical status, the initial rating of the esophageal examination results, and the treatment assignment. The reader reviewed and rated the endoscopic images of the esophageal examination from all the study sites. In the case of discrepancies between the original endoscopist's assessment and the central reader's assessment, the greatest abnormality was used for the analysis. In the case of the recording of video and/or photographic images being judged by the central reader to be an unsatisfactory low-quality recording (image is not sharp), the rating of the initial esophageal examination observation was documented and included only in the per protocol population.

Safety end points were AEs recorded from the start of the study until the end of the study; severity and relationship to treatment were recorded in patients' medical records and on the AE case report form. All SAEs were reported within 24 hours of notification of occurrence. In this study, patients were questioned using generalized and specific questions about clinical symptoms such as choking, throat pain, dysphagia, odynophagia, and esophageal pain per FDA recommendations for monitoring AEs. Electrocardiograms (12-lead electrocardiogram recorded at $25 \mathrm{~mm} / \mathrm{s}$ ) were monitored at baseline, whereas physical examination, vital signs, and clinical laboratory evaluations were monitored at baseline and visit 3 .

\section{Statistical analysis}

The sample size was expected to detect important drugrelated AEs. Setting the equivalence limit to be a difference in proportions of 0.2 , assuming that the rate of AEs within the control group would be $\sim 0.10$, and using 0.10 as an estimate for the proportion of patients with AEs within the treatment group, 30 patients per treatment arm would yield a power of $82 \%$ to show evidence of the treatment's equivalence to the control group at 0.05 significance level for common AEs. Calculations for this test were done using nQuery 6.01 (Statistical Solutions, Boston, MA, USA).

The tolerability population consisted of all patients who received $\geq 1$ dose of PEG 3350 ASC or placebo and had valid ratings from visual inspection of the oral cavity and endoscopic examination performed by a gastroenterologist and the central reader. In the case of discrepancies between assessments from the original endoscopist and the central reader, the grade corresponding to the greatest abnormality was used for analysis. A two-sample, noninferiority test for proportion of patients with abnormalities (Likert scale 1, 2, and 3) of the oral mucosa and esophageal mucosa was performed at the 0.05 significance level based on the tolerability population using the Cochran-Mantel-Haenszel chi-squared test. The safety population consisted of all patients who received $\geq 1$ dose of PEG 3350 or placebo and had $\geq 1$ postdose safety assessment. Treatment-emergent AEs (TEAEs) were summarized by treatment, severity, and relationship to treatment. A two-sample, noninferiority test for proportion of common AEs for PEG 3350 ASC to placebo was performed at the 0.05 significance level using the Cochran-Mantel-Haenszel chi-squared test.

The proportion of days with rescue medication was calculated as number of days on rescue medication/number of days treated with study medication $\times 100$.

\section{Results}

\section{Patient characteristics}

A total of 65 patients were enrolled in the study; 31 were randomized to PEG $3350 \mathrm{ASC}$ and 34 to placebo. There were 62 patients who completed the study; one patient each withdrew consent, was lost to follow-up, and was withdrawn because of a fractured wrist. The safety population comprised 65 patients, and the tolerability population comprised 62 patients (Figure 2). Patient demographics and baseline characteristics were well balanced between the two groups 


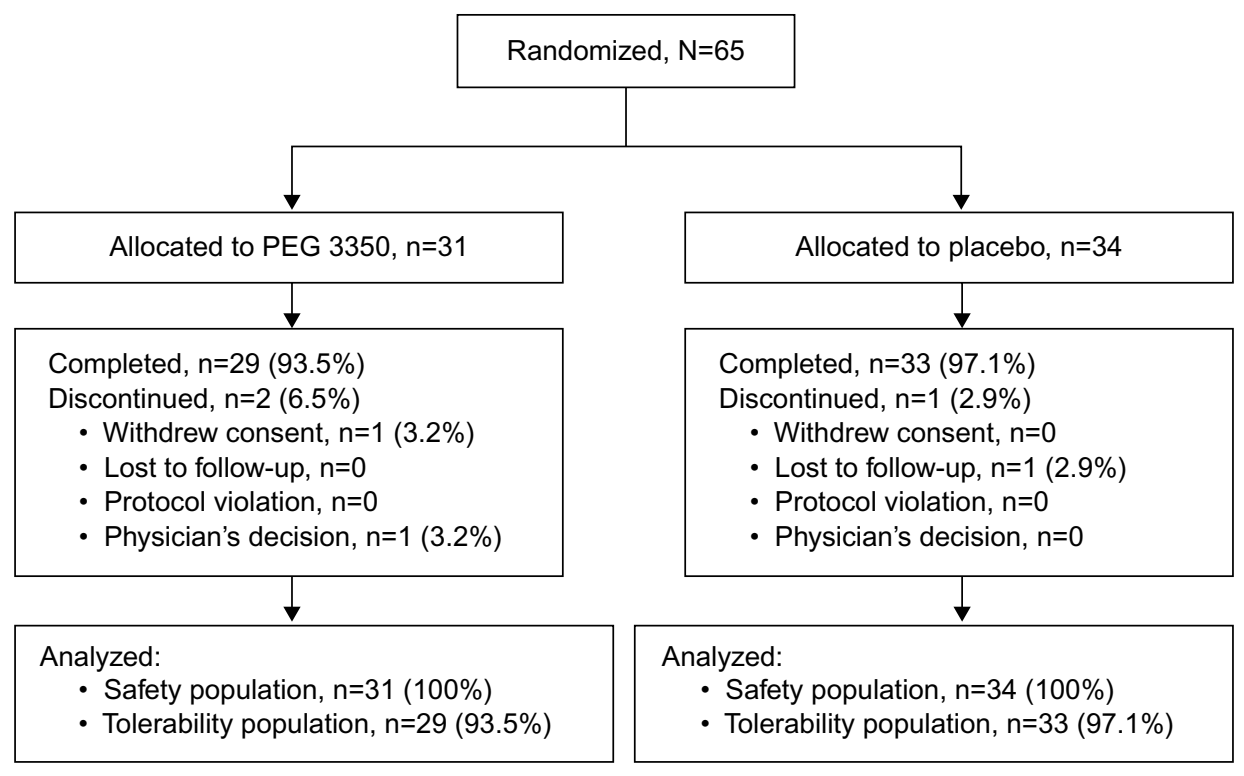

Figure 2 Disposition of patients.

Abbreviation: PEG, polyethylene glycol.

Table I Patient demographics

\begin{tabular}{|c|c|c|c|}
\hline \multirow[t]{2}{*}{ Characteristic } & \multicolumn{3}{|l|}{ Treatment } \\
\hline & $\begin{array}{l}\text { PEG } 3350 \\
(n=31)\end{array}$ & $\begin{array}{l}\text { Placebo } \\
(n=34)\end{array}$ & $\begin{array}{l}\text { Overall } \\
(\mathrm{N}=65)\end{array}$ \\
\hline Age (years), mean (SD) & $44.5(13.87)$ & $48(15.17)$ & $46.4(14.56)$ \\
\hline \multicolumn{4}{|l|}{ Sex, n (\%) } \\
\hline Male & $7(22.6)$ & $7(20.6)$ & $14(2 \mid .5)$ \\
\hline Female & $24(77.4)$ & $27(79.4)$ & $51(78.5)$ \\
\hline \multicolumn{4}{|l|}{ Race, n (\%) } \\
\hline White & $16(51.6)$ & $21(61.8)$ & 37 (56.9) \\
\hline Black or African American & $13(4 \mid .9)$ & II (32.4) & 24 (36.9) \\
\hline Other & $2(6.5)$ & $2(5.9)$ & $4(6.2)$ \\
\hline \multicolumn{4}{|l|}{ Ethnicity, n (\%) } \\
\hline Hispanic/Latino & $5(16.1)$ & $4(11.8)$ & $9(13.8)$ \\
\hline Non-Hispanic Latino & $26(83.9)$ & $30(88.2)$ & $56(86.2)$ \\
\hline
\end{tabular}

Abbreviations: $N$, number of patients; PEG, polyethylene glycol; SD, standard deviation.

(Table 1). There were no findings of concern in the medical history, baseline characteristics, or symptoms prior to dosing for any patients. All electrocardiograms performed on visit 1 were either normal or abnormal but not clinically significant.

\section{Tolerability outcome}

Upon visual inspection of the oral cavity, no patient in either treatment group exhibited inflammation of the oral mucosa during visit 2 (before treatment) or visit 3 (after treatment, Table 2). Fewer abnormalities of the esophageal mucosa were observed in the PEG 3350 ASC group than in the placebo group on visit 3, with no significant difference between treatment groups. Worst abnormality endoscopy data revealed incidences of mild inflammation of the esophageal mucosa
Table 2 Summary of oral cavity inspection and endoscopy examination

\begin{tabular}{|c|c|c|c|c|c|}
\hline \multirow[b]{2}{*}{ End point } & \multirow[b]{2}{*}{$\begin{array}{l}\text { Likert scale } \\
\text { score }^{\text {a }}\end{array}$} & \multicolumn{2}{|c|}{ PEG $3350(n=31)$} & \multicolumn{2}{|c|}{ Placebo $(n=34)$} \\
\hline & & $\begin{array}{l}\text { Visit } 2 \\
\text { n (\%) }\end{array}$ & $\begin{array}{l}\text { Visit } 3 \\
\text { n (\%) }\end{array}$ & $\begin{array}{l}\text { Visit } 2 \\
\text { n (\%) }\end{array}$ & $\begin{array}{l}\text { Visit } 3 \\
\text { n (\%) }\end{array}$ \\
\hline \multirow[t]{5}{*}{ Oral mucosa } & 0 & $31(100)$ & $29(93.5)$ & $34(100)$ & $33(97.1)$ \\
\hline & I & - & - & - & - \\
\hline & 2 & - & - & - & - \\
\hline & 3 & - & - & - & - \\
\hline & Not evaluated & - & $2(6.5)$ & - & I (2.9) \\
\hline Esophageal & 0 & $31(100)$ & $26(83.9)$ & $34(100)$ & $29(85.3)$ \\
\hline \multirow[t]{4}{*}{ mucosa } & I & - & $2(6.5)$ & - & I (2.9) \\
\hline & 2 & - & I (3.2) & - & $3(8.8)$ \\
\hline & 3 & - & - & - & - \\
\hline & Not evaluated & - & $2(6.5)$ & - & I (2.9) \\
\hline Worst & 0 & - & 22 (7I.0) & - & $24(70.6)$ \\
\hline \multirow[t]{4}{*}{ abnormality $^{b}$} & I & - & $5(16.1)$ & - & $5(14.7)$ \\
\hline & 2 & - & $2(6.5)$ & - & $4(11.8)$ \\
\hline & 3 & - & & - & - \\
\hline & Not evaluated & - & $2(6.5)$ & - & I (2.9) \\
\hline
\end{tabular}

Notes: Not evaluated are those patients who withdrew from the study before visit 3. aLikert scale score $0=$ no inflammation (no erythema and no erosion/ ulceration); I= mild inflammation (erythema without erosion/ulceration); $2=$ moderate inflammation (erythema with erosion); and $3=$ severe inflammation (erythema with ulceration). 'Worst abnormality is the worst grade between esophageal mucosa and endoscopy overread.

Abbreviation: PEG, polyethylene glycol.

that were similar $(n=5)$ for both treatment groups. Moderate inflammation was more frequent in the placebo group $(n=4$; $11.8 \%$ ) than in the PEG 3350 ASC group ( $n=2 ; 6.5 \%$, Table 2$)$.

\section{Safety}

Overall, 40 TEAEs were observed in 15 of 31 patients (48.4\%) treated with PEG 3350 ASC, and 41 TEAEs were 
observed in 19 of 34 patients (55.9\%) given placebo - nonsignificant difference of $-7.5 \%(95 \% \mathrm{CI}$ : $-21.3,6.3)$ between treatment groups (Table 3). Most frequently reported TEAEs were GI disorders (11 [35.5\%] for PEG 3350 and 15 [44.1\%]

Table 3 Frequency of treatment-emergent adverse events $(\geq 2$ patients) ${ }^{\mathrm{a}}$

\begin{tabular}{|c|c|c|c|}
\hline $\begin{array}{l}\text { System organ class } \\
\text { Preferred term }\end{array}$ & $\begin{array}{l}\text { PEG } 3350 \\
(n=3 I)\end{array}$ & $\begin{array}{l}\text { Placebo } \\
(n=34)\end{array}$ & $\begin{array}{l}\text { Overall } \\
(N=65)\end{array}$ \\
\hline Overall total, n (\%) & I 5 (48.4) [40] & $19(55.9)[4 \mathrm{I}]$ & $34(52.3)[8 I]$ \\
\hline $\begin{array}{l}\text { Gastrointestinal } \\
\text { disorders, } n(\%)\end{array}$ & II (35.5) [18] & $15(44.1)$ [24] & $26(40.0)[42]$ \\
\hline Abdominal pain, n (\%) & $4(12.9)[4]$ & $6(17.6)[7]$ & $10(15.4)[\mathrm{II}]$ \\
\hline Nausea, n (\%) & $4(12.9)[4]$ & $2(5.9)[2]$ & $6(9.2)[6]$ \\
\hline Flatulence, n (\%) & $I(3.2)[1]$ & $4(11.8)[4]$ & $5(7.7)[5]$ \\
\hline $\begin{array}{l}\text { Abdominal distension, } \\
\mathrm{n}(\%)\end{array}$ & - & $3(8.8)[3]$ & $3(4.6)[3]$ \\
\hline Diarrhea, n (\%) & $2(6.5)[2]$ & I (2.9) [I] & $3(4.6)[3]$ \\
\hline $\begin{array}{l}\text { Abdominal discomfort, } \\
\mathrm{n}(\%)\end{array}$ & - & $2(5.9)[2]$ & $2(3.1)[2]$ \\
\hline Hemorrhoids, n (\%) & $2(6.5)[2]$ & - & $2(3.1)[2]$ \\
\hline $\begin{array}{l}\text { Nervous system } \\
\text { disorders, } \mathrm{n}(\%)\end{array}$ & $8(25.8)[11]$ & $8(23.5)[10]$ & $16(24.6)[2 I]$ \\
\hline Headache, n (\%) & $6(19.4)[8]$ & $5(14.7)[6]$ & II (I6.9) [I4] \\
\hline Dizziness, n (\%) & $2(6.5)[2]$ & $2(5.9)[3]$ & $4(6.2)[5]$ \\
\hline $\begin{array}{l}\text { Metabolism and nutrition } \\
\text { disorders, } \mathrm{n}(\%)\end{array}$ & $\mathrm{I}(3.2)[\mathrm{I}]$ & $2(5.9)[2]$ & $3(4.6)[3]$ \\
\hline Decreased appetite, n (\%) & $\mathrm{I}(3.2)[\mathrm{I}]$ & $2(5.9)[2]$ & $3(4.6)[3]$ \\
\hline
\end{tabular}

Notes: Number of AEs given in square brackets. N, number of patients studied. a Patients may have $>I A E$ and may be counted more than once in each category. Abbreviations: $\mathrm{AE}$, adverse event; $\mathrm{PEG}$, polyethylene glycol.

Table 4 Analysis of TEAEs ${ }^{\mathrm{a}}$

\begin{tabular}{|c|c|c|c|}
\hline & $\begin{array}{l}\text { PEG } 3350 \\
(n=31)\end{array}$ & $\begin{array}{l}\text { Placebo } \\
(n=34)\end{array}$ & $\begin{array}{l}\text { Overall } \\
(\mathrm{N}=65)\end{array}$ \\
\hline $\begin{array}{l}\text { Patients with TEAE, } \\
\mathrm{n}(\%)\end{array}$ & $15(48.4)$ & $19(55.9)$ & $34(52.3)$ \\
\hline Number of TEAEs & 40 & 41 & 81 \\
\hline \multicolumn{4}{|l|}{ Intensity (all TEAEs) } \\
\hline Mild, n (\%) & $13(41.9)$ [35] & $19(55.9)[4 I]$ & $32(49.2)[76]$ \\
\hline Moderate, n (\%) & $5(16.1)[5]$ & - & $5(7.7)[5]$ \\
\hline Total, n (\%) & I5 (48.4) [40] & $19(55.9)[4 I]$ & $34(52.3)[8 I]$ \\
\hline \multicolumn{4}{|c|}{ Intensity (probably or definitely related) } \\
\hline Mild, n (\%) & $8(25.8)[14]$ & $14(4 \mid .2)[19]$ & $22(33.8)[33]$ \\
\hline Moderate, n (\%) & - & - & - \\
\hline Total, n (\%) & $8(25.8)[14]$ & $14(4 \mid .2)[19]$ & $22(33.8)[33]$ \\
\hline \multicolumn{4}{|l|}{ Relationship to study drug } \\
\hline Definitely related, $n(\%)$ & $I(3.2)[I]$ & $\mathrm{I}(2.9)[\mathrm{I}]$ & $2(3.1)[2]$ \\
\hline Probably related, $\mathrm{n}(\%)$ & $7(22.6)[13]$ & $14(4 \mid .2)[18]$ & $21(32.3)[31]$ \\
\hline Not related, $\mathrm{n}(\%)$ & $12(38.7)[26]$ & 13 (38.2) [22] & $25(38.5)[48]$ \\
\hline \multicolumn{4}{|c|}{ Relationship to rescue medication } \\
\hline Definitely related, n (\%) & - & - & - \\
\hline Probably related, n (\%) & - & - & - \\
\hline Not related, n (\%) & I5 (48.4) [40] & $19(55.9)[4 I]$ & $34(52.3)[8 I]$ \\
\hline
\end{tabular}

Notes: Number of AEs given in square brackets. $\mathrm{N}$, total number of patients

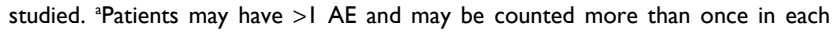
category.

Abbreviations: AE, adverse event; PEG, polyethylene glycol; TEAE, treatmentemergent adverse event. for placebo). GI disorders reported in the PEG 3350 group were nausea, abdominal pain, and diarrhea, whereas in the placebo group, they were abdominal pain, flatulence, and abdominal distension. Only one TEAE in each treatment group was considered definitely related to treatment; fewer TEAEs were considered probably related to PEG 3350 ASC $(13 / 40 ; 32.5 \%)$ than placebo $(18 / 41 ; 43.9 \%)$. All 41 TEAEs reported in the placebo group were mild in intensity, and of the 40 TEAEs reported in the PEG 3350 ASC group, 35 were mild and five were moderate in intensity (Table 4). No TEAE in either treatment group was considered related to rescue medication. No SAEs or deaths were reported, and no patient was discontinued from the study because of an AE. No postdose clinically significant findings were noted for vital signs, physical examinations, or clinical laboratory evaluations. No patients in the PEG 3350 group and four $(12 \%)$ in the placebo group took rescue medication (mean [standard deviation] of 1.7 [5.58] days).

\section{Discussion}

OTC laxatives are frequently used by patients to relieve constipation symptoms. ${ }^{2,20}$ The FDA has approved OTC use of PEG 3350 powder for solution once a day, for not more than a week, to treat occasional constipation. This has also benefited patients with chronic constipation. In the current study, a more convenient ASC of PEG 3350 was evaluated for its safety and tolerability in patients with chronic constipation. This ASC formulation, at its recommended dosage, would provide the same dose as the powder formulation; hence, the ASC was expected to be identical in efficacy and safety to the powder formulation. Results of this randomized, placebo-controlled study demonstrate that PEG 3350 ASC, ingested in its undiluted form, is generally safe and well tolerated in patients with functional constipation. The results are consistent with the known favorable safety and tolerability profile of the powder formulation of PEG $3350,{ }^{4}$ which has been previously demonstrated in multiple short-term ( 72 hours- 4 weeks) $)^{15,16,19}$ and long-term (6-12 months) studies. ${ }^{11,14}$

A pilot study that evaluated different doses $(51 \mathrm{~g}, 68 \mathrm{~g}$, and $85 \mathrm{~g}$ ) of PEG 3350 versus placebo for safety and efficacy over a 72-hour period showed that doses as high as $85 \mathrm{~g}$ were tolerated with no adverse reactions; no change in laboratory findings; and no complaints of cramps, diarrhea, or incontinence at any dose. This concurs with results from the present study and further substantiates the tolerability and safety of PEG 3350 ASC. ${ }^{12}$

Multiple randomized controlled trials have reiterated the clinical efficacy and excellent safety of PEG 3350 in small to 
large cohorts of patients with chronic constipation. Consistently across studies, PEG 3350 has proven to be effective, safe, and well tolerated with minor AEs that were mainly confined to GI disorders (eg, diarrhea, loose stools, flatulence, and nausea), which are not unexpected from a laxative. ${ }^{10-16}$ In the present study, GI disorders constituted $>50 \%$ of the AEs, with fewer incidences of abdominal pain and flatulence compared with placebo since PEG is not fermented by gut flora and does not contribute to gas production. ${ }^{8}$ No new AEs emerged with continued PEG 3350 exposure, reflective of the minimal absorption and inertness of PEG 3350.

Large volumes of PEG have been widely used for bowel cleansing prior to colonoscopy because of its efficacy and acceptable safety. ${ }^{21}$ A major concern of osmotic laxatives, however, is the ability to cause mucosal injuries. ${ }^{22}$ In an attempt to identify any mucosal abnormality that could arise from ingestion of the PEG 3350 aqueous concentrate in its undiluted form, unique end points (ie, examination of oral and esophageal mucosa by endoscopy) were included in the study. Inflammation of the oral mucosa was not observed in any patient administered PEG 3350 ASC, and similar abnormalities in esophageal mucosa occurred in each treatment group. These results extend previous observations that GI lavage containing PEG 3350 did not alter colonic mucosal histology ${ }^{23}$ and reaffirm the safety and tolerability of PEG 3350. Systemic absorption of PEG 3350 is minimal; once absorbed, the drug is excreted solely by the kidneys. Consistent with previous reports of minimal absorption and maximal excretion in feces, ${ }^{7}$ no clinically significant changes from baseline in renal function tests (ie, estimated glomerular filtration rate and serum creatinine level values) were observed after treatment, indicating that PEG 3350 ASC does not affect renal function.

In the present study, bisacodyl was allowed as a rescue medication to be taken in the absence of a BM for 72 hours after administration of treatment drug. Bisacodyl was taken by four patients in the placebo group, but none in the PEG 3350 ASC group, indicating that PEG 3350 ASC successfully managed patients' constipation symptoms, corroborating previous findings demonstrating that PEG 3350 is efficacious in treating multiple aspects of constipation.

The limitations of this study should be acknowledged. This study was not double blinded. The study was only blinded to the investigators. Though the study was not open-label, patients who had previously used PEG 3350 laxatives would be able to identify that placebo did not contain the active ingredient because of potential odor differences and mouth-feel differences inherent to PEG 3350.
An organoleptic evaluation of the undiluted concentrate was not undertaken since it would have provided data on an investigational drug that was not taken as directed for the product after marketing approval. However, an organoleptic evaluation of the product used as directed (in 4-8 ounces of liquid) should be conducted in future studies to compare palatability of PEG 3350 with currently available formulations. Another study limitation was the fewer number of patients recruited. Although no statistical analysis was performed, the demographics between PEG 3350 treatment and placebo groups in this study seemed to be comparable, indicating that the randomization was effective. Instructions for OTC usage of PEG 3350 for treatment of occasional constipation state that PEG 3350 should be taken for a week; however, in this study, safety and tolerability of PEG 3350 ASC were evaluated over a 14-day treatment period with a 1-month follow-up to assess potential treatment-related AEs. The extended treatment period and follow-up period are strengths of this study. Although large-scale studies are needed to confirm these findings, the present study identified no mucosal injury or major clinical AEs, indicating that PEG 3350 ASC is safe and well tolerated in its concentrated form. It should be reiterated that according to the directions for its intended use, PEG 3350 should be diluted before use.

\section{Conclusion}

Single daily doses of PEG 3350 ASC ingested in undiluted form were generally safe and well tolerated in patients with chronic constipation, and can be considered as a favorable option for short-term treatment.

\section{Acknowledgments}

Medical writing and/or editorial assistance was provided by Elphine Telles, PhD, of Cactus Communications. This assistance was funded by Merck \& Co., Inc., Kenilworth, NJ, USA.

\section{Author contributions}

McGraw T contributed to the conception, design, and planning of the study, data acquisition and analysis, interpretation of results, drafting the manuscript, and critically reviewing and revising the manuscript in terms of intellectual content.

\section{Disclosure}

Funding for this research was provided by Merck \& Co., Inc., Kenilworth, NJ, USA. McGraw T was an employee of Merck \& Co., Inc., Kenilworth, NJ, USA when the study was conducted. Merck \& Co. was involved in study design; in the collection, analysis, and interpretation of data; in the writing of the report; 
and in the decision to submit the article for publication. The author reports no other conflicts of interest in this work.

\section{References}

1. Higgins PD, Johanson JF. Epidemiology of constipation in North America: a systematic review. Am J Gastroenterol. 2004;99(4):750-759.

2. Wald A, Scarpignato C, Mueller-Lissner S, et al. A multinational survey of prevalence and patterns of laxative use among adults with self-defined constipation. Aliment Pharmacol Ther. 2008;28(7):917-930.

3. Suares NC, Ford AC. Prevalence of, and risk factors for, chronic idiopathic constipation in the community: systematic review and metaanalysis. Am J Gastroenterol. 2011;106(9):1582-1591. [quiz 1, 92].

4. Horn JR, Mantione MM, Johanson JF. OTC polyethylene glycol 3350 and pharmacists' role in managing constipation. J Am Pharma Assoc (2003). 2012;52(3):372-380.

5. Longstreth GF, Thompson WG, Chey WD, Houghton LA, Mearin F, Spiller RC. Functional bowel disorders. Gastroenterology. 2006;130(5):1480-1491.

6. NDA [webpage on the Internet]. Clinical Pharmacology and Biopharmaceutics Review. Center for Drug Evaluation and Research; 2006;NDA 22-015. Available from: http://www.accessdata.fda.gov/ drugsatfda_docs/nda/2006/022015s000_ClinPharmR.pdf. Accessed April 29, 2016.

7. Pelham RW, Nix LC, Chavira RE, Cleveland MV, Stetson P. Clinical trial: single- and multiple-dose pharmacokinetics of polyethylene glycol (PEG-3350) in healthy young and elderly subjects. Aliment Pharmacol Ther. 2008;28(2):256-265.

8. BouhnikY, Neut C, Raskine L, et al. Prospective, randomized, parallelgroup trial to evaluate the effects of lactulose and polyethylene glycol4000 on colonic flora in chronic idiopathic constipation. Aliment Pharmacol Ther. 2004;19(8):889-899.

9. Schiller LR, Emmett M, Santa Ana CA, Fordtran JS. Osmotic effects of polyethylene glycol. Gastroenterology. 1988;94(4):933-941.

10. Di Palma JA, Cleveland MV, McGowan J, Herrera JL. A randomized, multicenter comparison of polyethylene glycol laxative and tegaserod in treatment of patients with chronic constipation. Am J Gastroenterol. 2007;102(9):1964-1971.
11. Di Palma JA, Cleveland MV, McGowan J, Herrera JL. An open-label study of chronic polyethylene glycol laxative use in chronic constipation. Aliment Pharmacol Ther. 2007;25(6):703-708.

12. Di Palma JA, Smith JR, Cleveland M. Overnight efficacy of polyethylene glycol laxative. Am J Gastroenterol. 2002;97(7):1776-1779.

13. DiPalma JA, Cleveland MB, McGowan J, Herrera JL. A comparison of polyethylene glycol laxative and placebo for relief of constipation from constipating medications. South Med J. 2007;100(11):1085-1090.

14. Dipalma JA, Cleveland MV, McGowan J, Herrera JL. A randomized, multicenter, placebo-controlled trial of polyethylene glycol laxative for chronic treatment of chronic constipation. Am J Gastroenterol. 2007;102(7):1436-1441.

15. DiPalma JA, DeRidder PH, Orlando RC, Kolts BE, Cleveland MB. A randomized, placebo-controlled, multicenter study of the safety and efficacy of a new polyethylene glycol laxative. Am J Gastroenterol. 2000;95(2):446-450.

16. Cleveland MV, Flavin DP, Ruben RA, Epstein RM, Clark GE. New polyethylene glycol laxative for treatment of constipation in adults: a randomized, double-blind, placebo-controlled study. South Med J. 2001;94(5):478-481.

17. Migeon-Duballet I, Chabin M, Gautier A, et al. Long-term efficacy and cost-effectiveness of polyethylene glycol 3350 plus electrolytes in chronic constipation: a retrospective study in a disabled population. Curr Med Res Opin. 2006;22(6):1227-1235.

18. Zurad EG, Johanson JF. Over-the-counter laxative polyethylene glycol 3350: an evidence-based appraisal. Curr Med Res Opin. 2011;27(7):1439-1452.

19. Tran LC, Di Palma JA. Lack of lasting effectiveness of PEG 3350 laxative treatment of constipation. J Clin Gastroenterol. 2005;39(7):600-602.

20. Johanson JF, Kralstein J. Chronic constipation: a survey of the patient perspective. Aliment Pharmacol Ther. 2007;25(5):599-608.

21. Balaban DH, Thompson WO. Oral bowel preparation for colonoscopy. Aliment Pharmacol Ther. 2007;26(6):965-966. [author reply 966-967].

22. Lawrance IC, Willert RP, Murray K. Bowel cleansing for colonoscopy: prospective randomized assessment of efficacy and of induced mucosal abnormality with three preparation agents. Endoscopy. 2011;43(5):412-418.

23. Pockros PJ,Foroozan P. Golytely lavage versus a standard colonoscopy preparation. Effect on normal colonic mucosal histology. Gastroenterology. 1985;88(2):545-548
Clinical and Experimental Gastroenterology

\section{Publish your work in this journal}

Clinical and Experimental Gastroenterology is an international, peerreviewed, open access, online journal publishing original research, reports, editorials, reviews and commentaries on all aspects of gastroenterology in the clinic and laboratory. This journal is included on PubMed. The manuscript management system is completely online

\section{Dovepress}

and includes a very quick and fair peer-review system, which is all easy to use. Visit http://www.dovepress.com/testimonials.php to read real quotes from published authors. 